\title{
Intestinal ischemia-reperfusion induces the release of IL-17A to regulate cell inflammation, apoptosis and barrier damage
}

\author{
LI XIAO, WAN-HUA ZHANG, YIN HUANG and PENG HUANG \\ Department of Pediatrics, South Medical University Affiliated Maternal and \\ Child Health Hospital of Foshan, Foshan, Guangdong 528000, P.R. China
}

Received September 10, 2021; Accepted November 16, 2021

DOI: $10.3892 /$ etm.2021.11081

\begin{abstract}
Intestinal ischemia-reperfusion (I/R) injury promotes the release of IL-17A, and previous studies have indicated that TGF- $\beta$ activated kinase 1 (TAK1) is an important signaling molecule in the regulatory function of IL-17A. The present study aimed to explore the potential effects of IL-17A release in intestinal I/R injury, and to investigate the underlying regulatory mechanisms. Initially, the expression levels of TAK 1 and JNK in a hypoxia/reoxygenation model were determined, and the effects of TAK1-knockdown on JNK phosphorylation and the viability, inflammation, apoptosis and barrier function of Caco-2 cells were assessed using Cell Counting Kit-8, reverse transcription-quantitative PCR, TUNEL and transepithelial electrical resistance assays, respectively. Subsequently, an antibody targeting IL-17A was used, and the effects of the IL-17A antibody on the expression levels of TAK1 as well as cell viability, inflammation, apoptosis and barrier function were determined. The results of the present study demonstrated that TAK1-knockdown markedly reduced JNK phosphorylation and improved the levels of cell viability, inflammation, apoptosis and barrier function via the MAPK signaling pathway. In addition, treatment with the IL-17A antibody inhibited the expression of TAK1, and reversed the aforementioned effects of TAK1 on Caco- 2 cells. In conclusion, intestinal I/R induces the release of IL-17A to regulate cell viability, inflammation, apoptosis and barrier damage via the TAK1/MAPK signaling pathway.
\end{abstract}

\section{Introduction}

Following the advancement of shock treatment and the establishment and promotion of arterial bypass, thrombolytic

Correspondence to: Dr Peng Huang, Department of Pediatrics, South Medical University Affiliated Maternal and Child Health Hospital of Foshan, 11 Renmin West Road, Foshan, Guangdong 528000, P.R. China

E-mail: huangpenghp99@163.com

Key words: IL-17A, TGF- $\beta$ activated kinase 1, MAPK, JNK, intestinal ischemia-reperfusion therapy, extracorporeal circulation and organ transplantation in recent years, a variety of organs can receive reperfusion after ischemia (1-4). In the majority of cases, ischemia/reperfusion (I/R) can restore the function of organs and tissues and repair ischemic damage. As blood flow is often restored following ischemia, ischemic damage may be reduced; however, the dysfunction or structural damage caused may be aggravated, which is known as I/R injury (5). Thus, I/R injury is a common injury in surgical practice that affects a number of tissues and organs. It plays a notable role in the pathophysiological evolution of serious infections, trauma, shock, cardiopulmonary insufficiency, organ transplantation and stroke (6). Moreover, the intestine is an organ that is both fragile and sensitive; thus, it remains vulnerable to the development of severe I/R injury (7). The mechanisms underlying intestinal I/R injury are relatively complex, involving apoptosis, inflammation, oxidative stress, calcium overload and leukocyte adhesion (8). The current treatment of intestinal I/R injury depends on ischemic preconditioning; that is, exposing the tissue to a state of transient ischemia in advance (9). However, due to the unpredictability of intestinal I/R, ischemic preconditioning has encountered notable limitations in clinical practice (10).

The intestinal mucosal barrier is a key barrier for the body's defense against intestinal I/R injury. Under stress, the intestinal mucosal barrier is damaged and the permeability increases, which further aggravates the primary disease and causes multiple organ failure (11). Therefore, studying the regulation mechanism underlying the intestinal epithelial barrier under stress conditions will aid in the development of early prevention methods, and reduce the mortality of critically ill patients (12).

TGF- $\beta$ activated kinase 1 (TAK1), an important intermediate in a variety of innate immune signaling pathways (such as Toll-like receptor signaling), has been revealed to play a notable role in maintaining intestinal homeostasis (13). Results of a previous study revealed that TAK1 mediates lipopolysaccharide-induced $\mathrm{NF}-\kappa \mathrm{B}$ signal activation, and increases the permeability of intestinal tight junctions (14). In addition, results of a further study demonstrated that intestinal $\mathrm{I} / \mathrm{R}$ injury promotes the release of IL-17A (also known as IL-17) (15), and TAK1 has been identified as a key signaling molecule in the regulatory function of IL-17 $(16,17)$. Moreover, results of a previous study demonstrated that TAK1 activates the expression of downstream MAPK signaling proteins in acute kidney injury (18). 
To the best of our knowledge, the role of TAK 1 in intestinal $\mathrm{I} / \mathrm{R}$ injury is yet to be fully elucidated. Caco- 2 cells are a type of human clonal adenocarcinoma cells, whose structure and function are similar to differentiated small intestinal epithelial cells, and are recognized for use in in vitro research on the intestine (19). Therefore, using Caco-2 cells, the present study aimed to explore the effects of IL-17 release in intestinal I/R injury, and investigate the underlying regulatory mechanisms. The findings of the present study provide a theoretical basis for further study of IL-17, and contribute to further understanding the mechanisms underlying intestinal I/R injury.

\section{Materials and methods}

Cell culture and grouping. Caco-2 cells were obtained from Procell Life Science \& Technology Co., Ltd. Caco-2 cells were cultured in DMEM (Gibco; Thermo Fisher Scientific, Inc.) supplemented with $20 \%$ FBS (Gibco; Thermo Fisher Scientific, Inc.) and $1 \%$ penicillin/streptomycin, and maintained in an incubator containing $5 \% \mathrm{CO}_{2}$ at $37^{\circ} \mathrm{C}$. Cells in the normoxic incubator were regarded as the control group.

Caco-2 cells $\left(3 \times 10^{5} /\right.$ well) were seeded into six-well plates 1 day before transfection until $\sim 80 \%$ confluence was reached. Cells were subsequently transfected with $1 \mu \mathrm{g}$ pcDNA3.1-TAK1 vector (ov-TAK1), empty vector (ov-NC) (each, Hanbio Biotechnology Co., Ltd.), short hairpin (sh) RNAs against TAK1 (shRNA-TAK1; 5'-CCCGTGTGAACC ATCCTAATA-3') or a nonspecific sequence as negative control (shRNA-NC; 5'-CAACAAGATGAAGAGCACCAA-3') (each, Shanghai GenePharma Co., Ltd.) using Lipofectamine ${ }^{\circledR} 2000$ (Invitrogen; Thermo Fisher Scientific, Inc.) at $37^{\circ} \mathrm{C}$, according to the manufacturer's protocol. Following transfection for $24 \mathrm{~h}$, the cells were used for subsequent experiments. These cells were regarded as the shRNA-NC/TAK1 or ov-NC/TAK1 groups.

Anisomycin, a type of JNK agonist, was purchased from GlpBio Technology and diluted to $4 \mu \mathrm{M}$. IL-17A neutralizing antibody (cat. no. AF-317) and IgG (cat. no. AB-108-C) were purchased from R\&D Systems, Inc. and diluted to $2 \mu \mathrm{g} / \mathrm{ml}$. Caco-2 cells treated with anisomycin or the aforementioned antibodies for $1 \mathrm{~h}$ at $37^{\circ} \mathrm{C}$ were used as the anisomycin/IgG/IL-17 antibody group.

To establish the hypoxia/reoxygenation (H/R) model, which mimicked the I/R model in vitro (20), the aforementioned groups of Caco-2 cells were transferred into an incubator containing $94 \% \mathrm{~N}_{2}, 5 \% \mathrm{CO}_{2}$ and $1 \% \mathrm{O}_{2}$ at $37^{\circ} \mathrm{C}$ to simulate hypoxia for $12 \mathrm{~h}$, and reoxygenated under normoxic conditions at $37^{\circ} \mathrm{C}$ for a further $6 \mathrm{~h}$. Cells that had undergone hypoxia and reoxygenation were labelled as the $\mathrm{H} / \mathrm{R}(+)$ group.

Reverse transcription-quantitative $(R T-q) P C R$. Total RNA was extracted from Caco-2 cells using TRIzol ${ }^{\circledR}$ reagent (Invitrogen; Thermo Fisher Scientific, Inc.), and subsequently reverse transcribed into cDNA using a universal reverse transcription kit (Beijing Baiao Laibo Technology Co., Ltd.) according to the manufacturer's instructions. The mRNA expression levels were measured using QuantiTect SYBR ${ }^{\circledR}$ Green PCR kit (Qiagen, Inc.) on a StepOnePlus ${ }^{\mathrm{TM}}$ Real-time PCR system (Applied Biosystems; Thermo Fisher Scientific, Inc.). The thermocycling conditions were as follows: Initial denaturation at $95^{\circ} \mathrm{C}$ for $3 \mathrm{~min}$; followed by 40 cycles of denaturation at $95^{\circ} \mathrm{C}$ for $30 \mathrm{sec}$, annealing at $60^{\circ} \mathrm{C}$ for $30 \mathrm{sec}$ and extension at $72^{\circ} \mathrm{C}$ for $30 \mathrm{sec}$. GAPDH was used as the internal reference and the relative mRNA expression levels were calculated using the $2^{-\Delta \Delta \mathrm{Cq}}$ method (21). The sequences of primer are listed in Table I.

Western blotting. Total proteins were extracted from Caco-2 cells using RIPA lysis buffer (Beyotime Institute of Biotechnology). The protein samples were determined by the BCA method and separated ( $25 \mu \mathrm{g} / \mathrm{lane})$ via SDS-PAGE on a 10 or $12 \%$ gel, and subsequently transferred onto PVDF membranes. Following blocking with 5\% skimmed milk for $1.5 \mathrm{~h}$ at room temperature, the membranes were incubated with primary antibodies against TAK1 (1:1,000; cat. no. ab109526), JNK (1:1,000; cat. no. ab76125), phosphorylated (p)-JNK (1:1,000; cat. no. ab124956), inducible nitric oxide synthase (iNOS; 1:1,000; cat. no. ab178945), cytochrome C oxidase subunit 2 (Cox 2 ; 1:1,000; cat. no. ab179800), cleaved caspase 3 (1:500; cat. no. ab2302), cleaved caspase 9 (1:200; cat. no. ab2324), occludin (1:1,000; cat. no. ab216327), claudin 1 (1:2,000; cat. no. ab211737), ZO-1 tight junction protein (ZO-1; 1:1,000; cat. no. ab216880), IL-17A (1:500; cat. no. ab79056) or GAPDH (1:1,000; cat. no. ab8245) (all Abcam) at $4^{\circ} \mathrm{C}$ overnight. Following primary incubation, the membranes were washed thrice with TBS-0.01\% Tween-20 for 10 min each and then incubated with a goat anti-rabbit HRP-conjugated secondary antibody (1:5,000; cat. no. ab97080; Abcam) for $2 \mathrm{~h}$ at room temperature. Protein bands were visualized using an ECL kit (cat. no. P0018AM; Beyotime Institute of Biotechnology), and the gray values were measured using ImageJ software (version 1.8; National Institutes of Health).

Cell Counting Kit-8 (CCK-8) assay. Caco-2 cells $\left(5 \times 10^{3}\right.$ cells/well) were cultured in 96 -well plates in the normoxic incubator for $24 \mathrm{~h}$, and $10 \mu \mathrm{l} \mathrm{CCK}-8$ reagent (Beyotime Institute of Biotechnology) was added to each well. Cells were subsequently incubated at $37^{\circ} \mathrm{C}$ for a further $2 \mathrm{~h}$. The absorbance of each well was measured using a microplate reader (Molecular Devices, LLC) at a wavelength of $450 \mathrm{~nm}$.

TUNEL assay. Caco- 2 cells $\left(2 \times 10^{4}\right.$ cells/well) were seeded into a 24-well plate and the TUNEL assay was performed using a TUNEL kit (cat.no. C1086; Beyotime Institute of Biotechnology), according to the manufacturer's instructions. Briefly, cells were fixed with $4 \%$ paraformaldehyde for $30 \mathrm{~min}$ at room temperature, and subsequently incubated with PBS containing $0.3 \%$ Triton $\mathrm{X}-100$ for $5 \mathrm{~min}$ at room temperature. Following the addition of TUNEL working fluid, cells were incubated for a further $1 \mathrm{~h}$ at $37^{\circ} \mathrm{C}$ in the dark. The nuclei was counterstained with DAPI for $10 \mathrm{~min}$ at room temperature. The results were observed at five random fields of view using a fluorescence microscope (magnification, x200; Olympus Corporation).

Transepithelial electrical resistance (TEER) assay. The TEER assay was used to determine the levels of barrier function. Caco- 2 cells $\left(3 \times 10^{4}\right)$ were seeded into the upper chamber of 24-well Transwell system plates (pore size, $0.4 \mu \mathrm{m}$; Corning, Inc.) and incubated with DMEM for 20 days at $37^{\circ} \mathrm{C}$. TEER was measured daily using an epithelial volt ohmmeter. The 
Table I. Primer sequences used for reverse transcriptionquantitative PCR analysis.

\begin{tabular}{ll} 
Gene & \multicolumn{1}{c}{ Sequence (5'-3') } \\
\hline TAK1 forward & ATGCGGTACTTTCCAGGAGC \\
TAK1 reverse & CTGTCCGTTGCCTGTGGTT \\
TNF- $\alpha$ forward & CACCACTTCGAAACCTGGGA \\
TNF- $\alpha$ reverse & AGGAAGGCCTAAGGTCCACT \\
IL-6 forward & CTTCGGTCCAGTTGCCTTCTC \\
IL-6 reverse & GGCATTTGTGGTTGGGTCAG \\
IL-1 $\beta$ forward & CTGAGCTCGCCAGTGAAATG \\
IL-1 $\beta$ reverse & TGTCCATGGCCACAACAACT \\
IL-17A forward & AACCGATCCACCTCACCTTG \\
IL-17A reverse & TCTCTTGCTGGATGGGGACA \\
GAPDH forward & GACTCATGACCACAGTCCATGC \\
GAPDH reverse & AGAGGCAGGGATGATGTTCTG
\end{tabular}

TAK1, TGF- $\beta$ activated kinase 1 .

calculation used was as follows: TEER $=\left(R_{1}-R_{2}\right) \times M . R_{1}$ represents the background resistance, $R_{2}$ represents the collagen layer and membrane insert resistance and $M$ represents the insert membrane area.

Statistical analysis. All experiments were performed at least three times. Data are presented as the mean \pm standard deviation, and statistical analysis was performed using GraphPad Prism version 8.0 (GraphPad Software, Inc.). Comparisons between multiple groups was performed using one-way ANOVA followed by a Tukey's post hoc test, and unpaired Student's t-tests were used for comparisons between two groups. $\mathrm{P}<0.05$ was considered to indicate a statistically significant difference.

\section{Results}

TAK1 inhibits JNK phosphorylation, and TAK1 knockdown improves cell viability and alleviates the inflammation of H/R-induced Caco-2 cells. The expression levels of TAK1 in the control and H/R groups were determined using RT-qPCR and western blotting. The results indicated that TAK1 expression was significantly upregulated in the H/R group compared with the control group (Fig. 1A and B). Moreover, the expression levels of p-JNK and JNK in the control and H/R groups were also assessed using western blotting. The expression level of p-JNK was significantly upregulated in the H/R group compared with the control group, whereas no marked difference was observed for the expression of JNK, suggesting that H/R may promote JNK phosphorylation (Fig. 1C). Subsequently, two shRNAs targeting TAK1 were transfected into Caco-2 cells, and the expression levels of TAK1 were subsequently assessed using RT-qPCR and western blotting. The expression levels of TAK 1 were reduced to a greater extent following transfection with shRNA-TAK1-1 compared with shRNA-TAK1-2; thus, cells transfected with shRNA-TAK1-1 were used in subsequent experiments (Fig. 1D and E).
In order to study the regulatory effects of TAK1 on the MAPK signaling protein JNK, a JNK agonist was used to treat the cells, and Caco- 2 cells were divided into five groups: i) Control; ii) $\mathrm{H} / \mathrm{R}$; iii) $\mathrm{H} / \mathrm{R}+$ shRNA-NC; iv) $\mathrm{H} / \mathrm{R}$ + shRNA-TAK1; and v) H/R + shRNA-TAK1 + anisomycin. Firstly, the effects of TAK1 on JNK expression were evaluated using western blotting, and the results revealed that TAK1 knockdown induced the significant upregulation of p-JNK expression following H/R. Moreover, anisomycin treatment significantly reversed the effects of TAK1 knockdown on p-JNK expression (Fig. 1F).

Cell viability was subsequently measured in the aforementioned groups using a CCK-8 assay. The results demonstrated that TAK1 knockdown significantly increased cell viability following H/R compared with the H/R + shRNA-NC group, while anisomycin reduced the level of cell viability after knockdown of TAK1 (Fig. 2A).

Furthermore, the expression levels of inflammatory factors were measured in the aforementioned groups using RT-qPCR and western blotting. Results of the present study demonstrated that TAK1 knockdown significantly reduced the expression levels of TNF- $\alpha$, IL- 6 and IL- $1 \beta$ compared with the H/R + shRNA-NC group, while treatment with anisomycin significantly reversed this reduction in expression levels (Fig. 2B). Results of western blotting demonstrated that TAK1 knockdown significantly reduced the expression levels of iNOS and Cox 2 to varying degrees compared with the H/R + shRNA-NC group, while treatment with anisomycin returned the expression levels to a level similar to those observed under H/R conditions (Fig. 2C). These results suggested that TAK1 knockdown may improve cell viability and alleviate inflammation in Caco-2 cells subjected to $\mathrm{H} / \mathrm{R}$ via inhibiting MAPK signal activation.

TAK1 knockdown alleviates the apoptosis and barrier dysfunction of H/R-induced Caco-2 cells by inhibiting MAPK signal activation. The effects of TAK1 knockdown on apoptosis and barrier dysfunction were evaluated. Apoptosis was assessed using TUNEL assays and western blotting. Results of the present study demonstrated that TAK1 knockdown led to a significant reduction in the intensity of green fluorescence emitted by apoptotic cells compared with the H/R + shRNA-NC group, indicating that TAK1 knockdown inhibited apoptosis. Moreover, treatment with anisomycin significantly increased the levels of apoptosis in the TAK 1 knockdown cells (Fig. 3A). Results of the western blotting also revealed that the expression levels of cleaved caspase 9 and cleaved caspase 3 were significantly reduced following TAK1 knockdown compared with the H/R + shRNA-NC groups, whereas treatment with anisomycin increased the expression levels of both cleaved caspase 9 and cleaved caspase 3 (Fig. 3B). Moreover, cell barrier function was assessed using TEER and western blotting. Results of the TEER assay indicated that TAK1 knockdown significantly reversed the H/R-mediated reduction in TEER value, while treatment of TAK1 knockdown cells with anisomycin reduced the TEER value (Fig. 3C). Furthermore, the expression levels of occludin, claudin-1 and ZO-1 were notably increased by TAK1 knockdown compared with those of the H/R + shRNA-NC group, and anisomycin treatment significantly reduced the expression levels of the aforementioned proteins (Fig. 3D). 
A

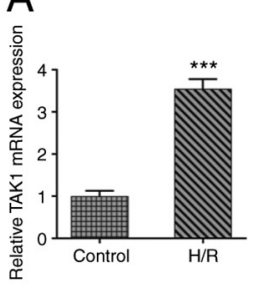

$B$
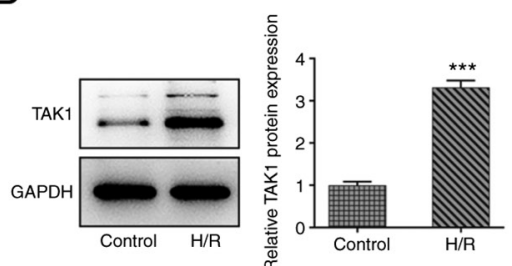

E

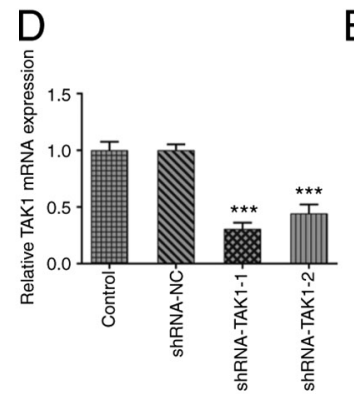

C

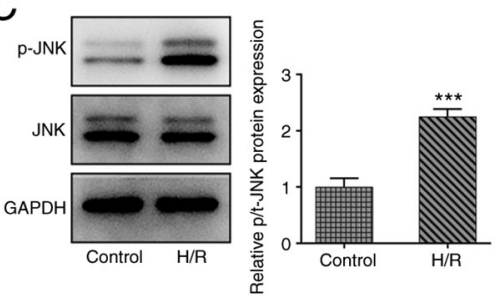

F

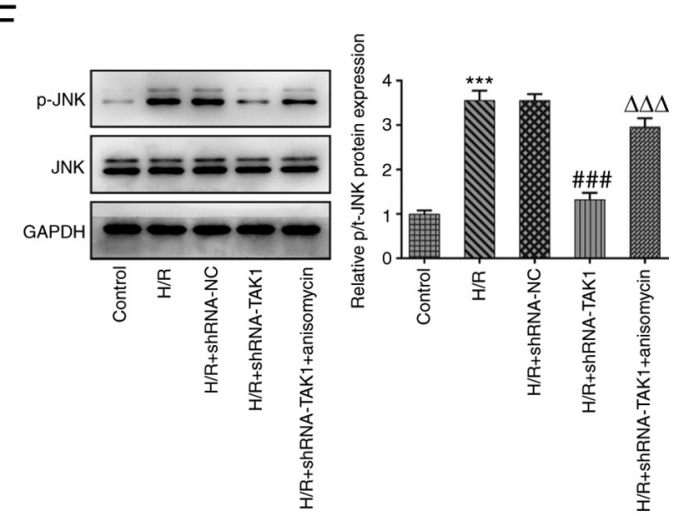

Figure 1. TAK1 inhibits JNK phosphorylation in H/R-induced Caco-2 cells. Expression levels of TAK1 in the control and H/R groups were determined using (A) RT-qPCR and (B) western blotting. (C) Expression levels of p-JNK and JNK were assessed using western blotting. Expression levels of TAK1 in the transfected cells were assessed using (D) RT-qPCR and (E) western blotting. (F) Expression levels of p-JNK and JNK in the five groups were evaluated using western blotting. ${ }^{* * *} \mathrm{P}<0.001$ vs. control/shRNA-NC; ${ }^{\# \# \#} \mathrm{P}<0.001$ vs. H/R + shRNA-NC; ${ }^{\Delta \Delta \Delta} \mathrm{P}<0.001$ vs. H/R + shRNA-TAK1. TAK1, TGF- $\beta$ activated kinase 1 ; H/R, hypoxia/reoxygenation; RT-qPCR, reverse transcription-quantitative PCR; p-, phosphorylated; shRNA, short hairpin RNA; NC, negative control.

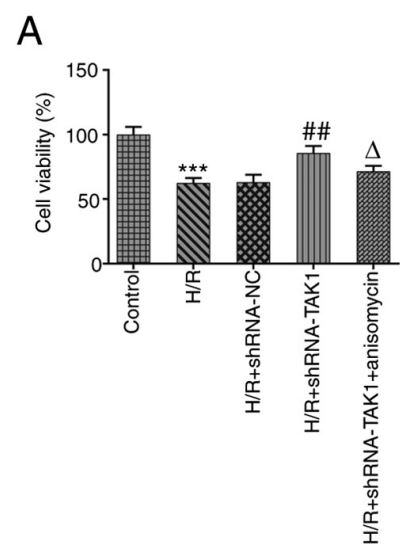

C

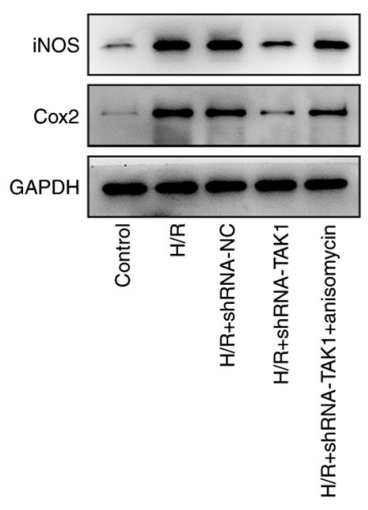

$B$ 亳

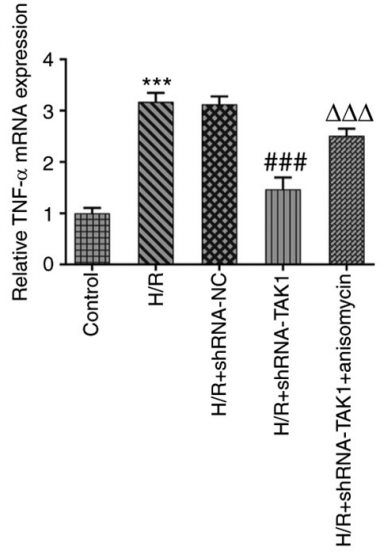

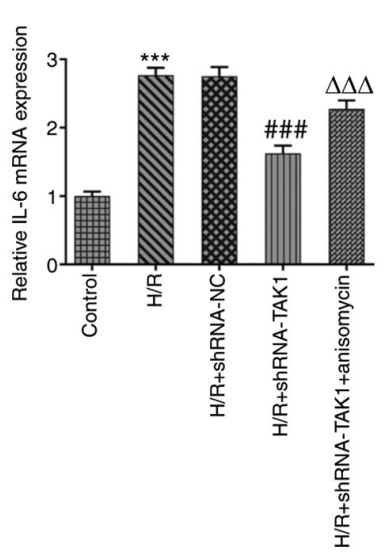

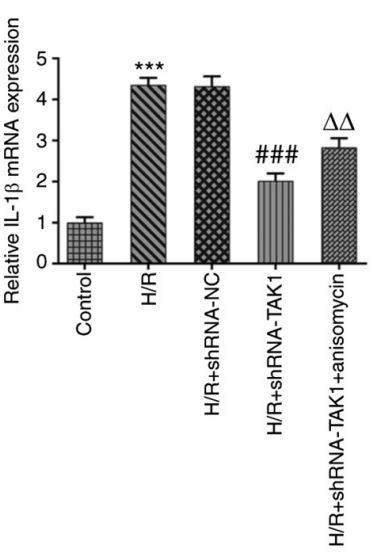

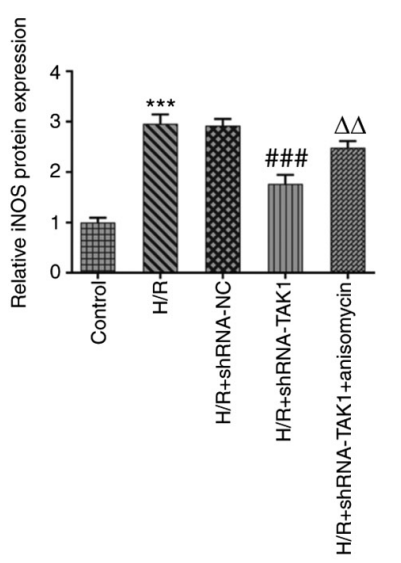

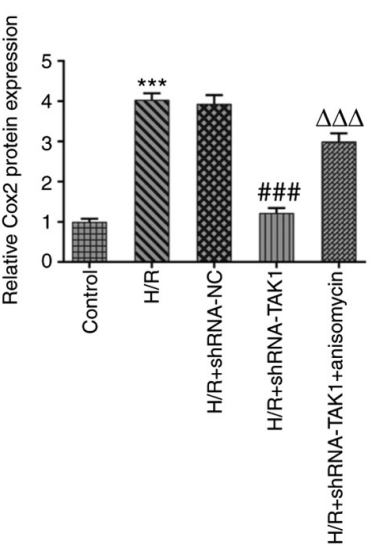

Figure 2. TAK1 knockdown improves cell viability and alleviates the inflammation of H/R-induced Caco-2 cells. (A) Cell viability in the five groups were measured using a Cell Counting Kit-8 assay. (B) Expression levels of TNF- $\alpha$, IL-6 and IL-1 $\beta$ in the five groups were determined using reverse transcription-quantitative PCR. (C) Expression levels of iNOS and Cox2 were determined western blotting. ${ }^{* * *} \mathrm{P}<0.001$ vs. control; ${ }^{\# \#} \mathrm{P}<0.01$, ${ }^{\# \#} \mathrm{P}<0.001 \mathrm{vs}$. H/R + shRNA-NC; ${ }^{\Delta} \mathrm{P}<0.05,{ }^{\Delta \Delta} \mathrm{P}<0.01,{ }^{\Delta \Delta \Delta} \mathrm{P}<0.001$ vs. H/R + shRNA-TAK1. TAK1, TGF- $\beta$ activated kinase $1 ; \mathrm{H} / \mathrm{R}$, hypoxia/reoxygenation; shRNA, short hairpin RNA; $\mathrm{NC}$, negative control; iNOS, inducible nitric oxide synthase; Cox 2 , cytochrome $\mathrm{C}$ oxidase subunit 2 . 
A
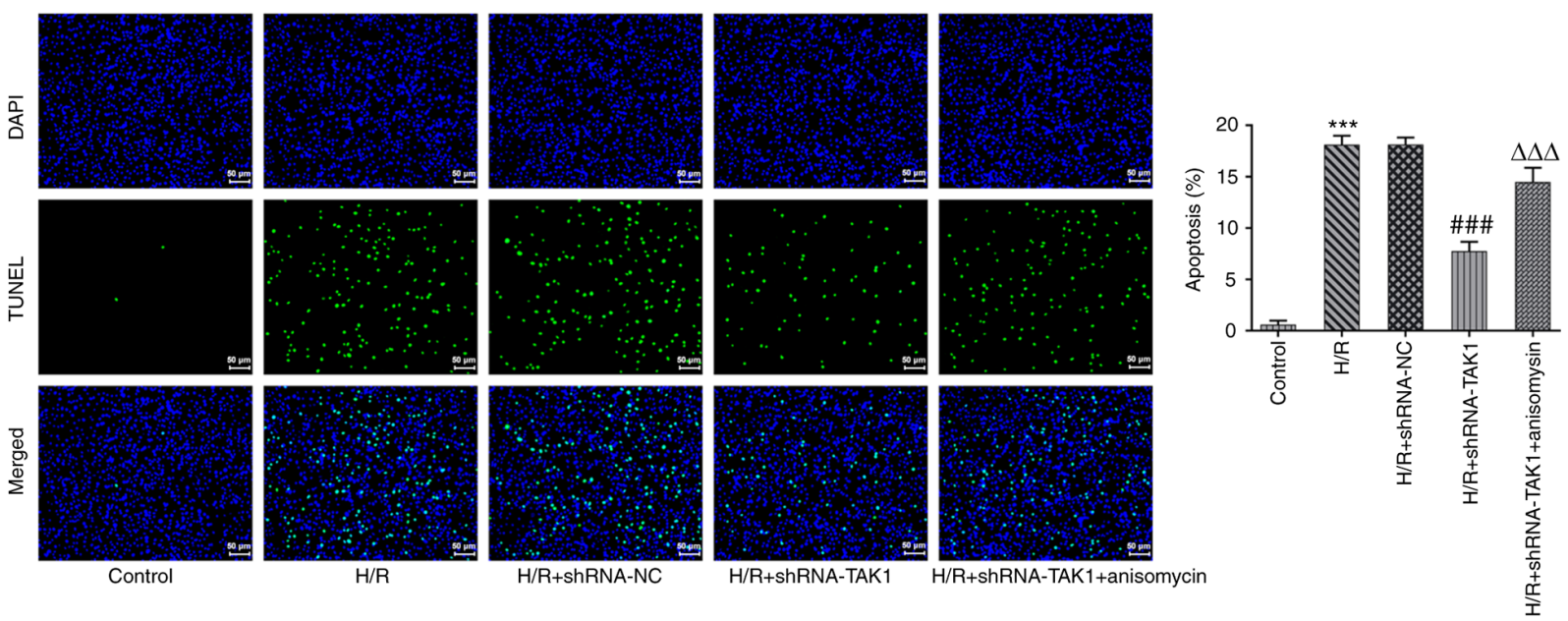

$\mathrm{B}$
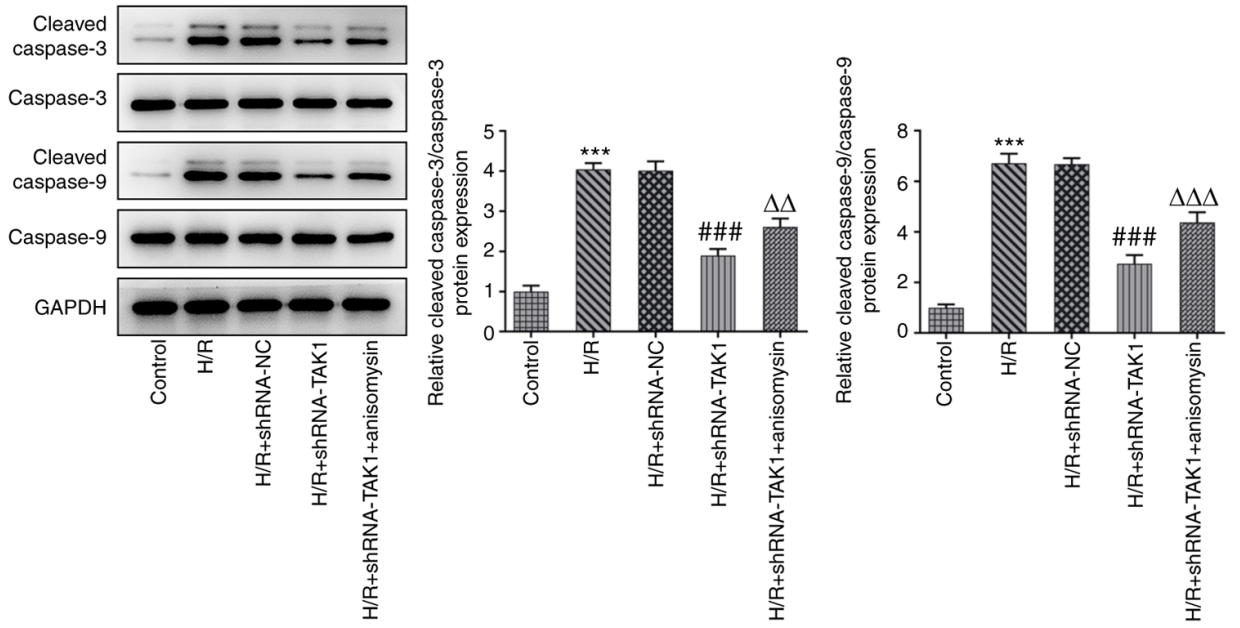

C
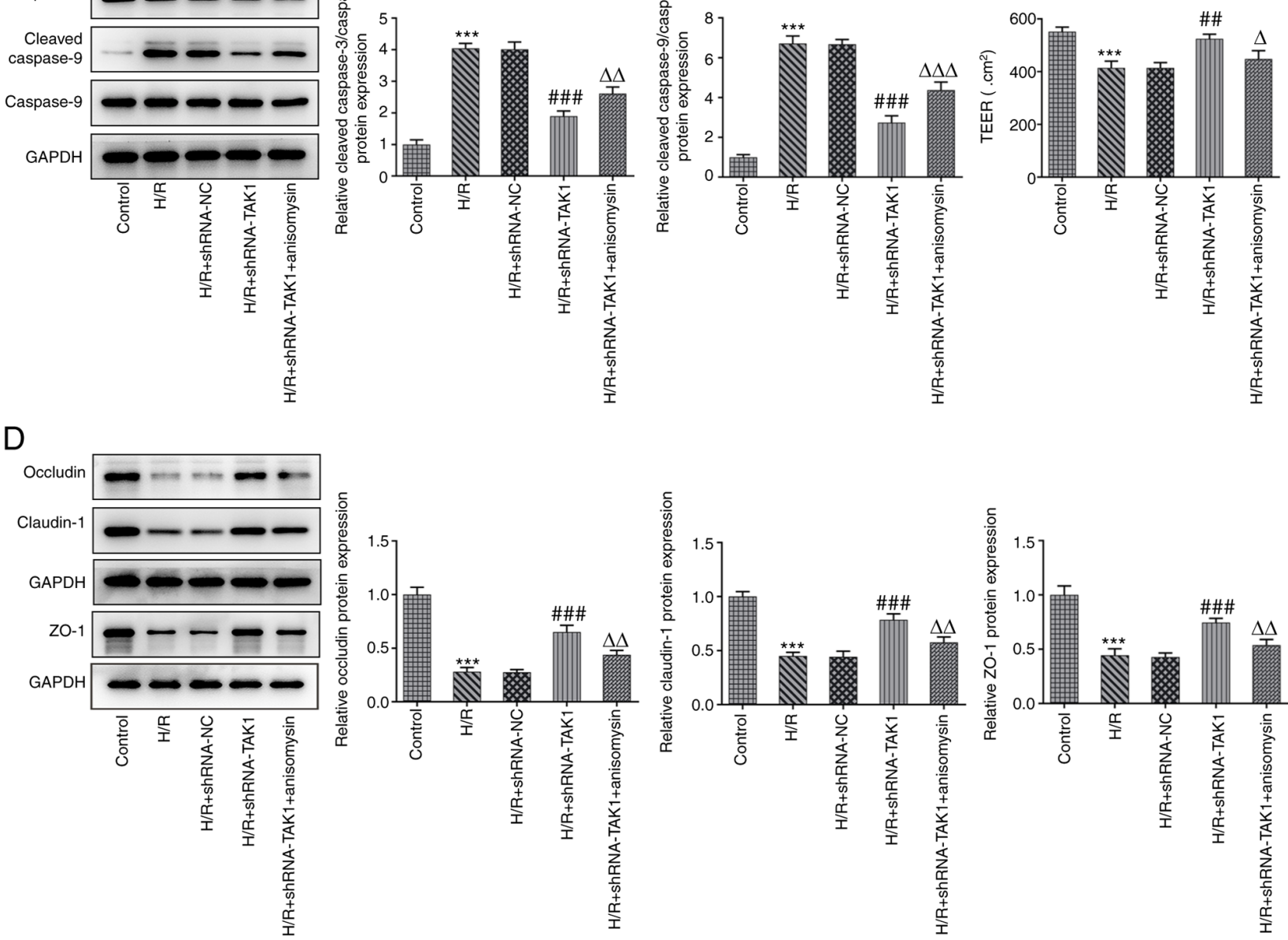

Figure 3. TAK1 knockdown alleviates the apoptosis and barrier dysfunction of H/R-induced Caco-2 cells via by inhibiting MAPK signal activation. (A) Apoptosis was assessed using a TUNEL assay (magnification, x200). (B) Expression levels of cleaved caspase 9 and cleaved caspase 3 were assessed using western blotting. (C) Cell barrier function was assessed using a transepithelial electrical resistance assay. (D) Expression levels of occludin, claudin-1 and ZO-1 were assessed using western blot analysis. ${ }^{* * *} \mathrm{P}<0.001$ vs. control; ${ }^{\# \#} \mathrm{P}<0.01,{ }^{\# \# \#} \mathrm{P}<0.001 \mathrm{vs}$. H/R + shRNA-NC; ${ }^{\Delta} \mathrm{P}<0.05,{ }^{\Delta \Delta} \mathrm{P}<0.01,{ }^{\Delta \Delta \Delta} \mathrm{P}<0.001$ vs. H/R + shRNA-TAK1. ZO-1, ZO-1 tight junction protein; TAK1, TGF-B activated kinase 1; H/R, hypoxia/reoxygenation; shRNA, short hairpin RNA; NC, negative control.

IL-17 antibody improves cell viability and alleviates the inflammation of $H / R$-induced Caco-2 cells via a reduction in TAK1 expression. Results of the present study demonstrated that TAK1 knockdown alleviated cell inflammation, apoptosis and dysfunction caused by intestinal I/R by regulating MAPK signaling; however, whether TAK1 participates in the IL-17 signaling pathway remains to be fully elucidated. Thus, the expression levels of IL-17 in the H/R group were initially 
determined using western blotting and RT-qPCR. Results of the present study revealed that IL-17 was highly expressed in cells that had undergone H/R. Subsequently, the expression levels of TAK1 in the H/R and H/R + IL-17 antibody groups were assessed using western blotting and RT-qPCR. TAK1 expression was significantly increased in the $H / R$ group compared with the control group (Fig. 4A and B), and TAK1 expression was markedly reduced in the H/R + IL-17 antibody group compared with the H/R group (Fig. 4C and D). These results indicated that the IL-17 antibody may reduce the expression of TAK1 by neutralizing secreted IL-17. Following the verification of high TAK1 expression levels in the transfected cells using western blotting and RT-qPCR (Fig. 4E and F), cells were divided into six groups: i) Control; ii) $\mathrm{H} / \mathrm{R}$; iii) $\mathrm{H} / \mathrm{R}+\mathrm{IgG}$; iv) $\mathrm{H} / \mathrm{R}+\mathrm{IL}-17$ antibody; v) $\mathrm{H} / \mathrm{R}+\mathrm{IL}-17$ antibody + ov-NC; and vi) $\mathrm{H} / \mathrm{R}+\mathrm{IL}-17$ antibody + ov-TAK1. Cell viability in the aforementioned six groups was measured using a CCK-8 assay. The results demonstrated that the addition of the IL-17 antibody significantly increased the levels of cell viability compared with $\mathrm{H} / \mathrm{R}+\mathrm{IgG}$, while the viability was significantly reduced in the H/R + IL-17 + ov-TAK1 group (Fig. 4G). In addition, the mRNA expression levels of TNF- $\alpha$, IL- 6 a and IL-1 $\beta$, and the protein expression levels of iNOS and Cox 2 were determined using RT-qPCR and western blotting, respectively. Results of the present study demonstrated that treatment with $H / R+$ IL-17 antibody significantly reduced the levels of inflammatory factors and inflammation-associated enzymes compared with the $\mathrm{H} / \mathrm{R}+\mathrm{IgG}$ group, whereas transfection with ov-TAK1 (H/R + IL-17 + ov-TAK1) significantly increased the IL-17 antibody-mediated reduced expression levels (Fig. 4H and I).

IL-17A antibody alleviates the apoptosis and barrier dysfunction of H/R-induced Caco-2 cells via a reduction in TAK1 expression. As the previously described methods focused on determining the effects of TAK1 knockdown on apoptosis and barrier function, the effects of the IL-17 antibody on apoptosis and barrier function were also investigated. The results of the TUNEL assay demonstrated that treatment with the IL-17 antibody significantly reduced the fluorescence emitted by apoptotic cells compared with the $\mathrm{H} / \mathrm{R}+\mathrm{IgG}$ group, whereas transfection with ov-TAK1 increased this fluorescence compared with the H/R + IL-17 antibody + ov-NC group (Fig. 5A). Moreover, the results of western blotting revealed that treatment with the IL-17 antibody significantly decreased the expression levels of cleaved caspase 9 and cleaved caspase 3 compared with the $\mathrm{H} / \mathrm{R}+\mathrm{IgG}$ group, whereas transfection with ov-TAK1 reversed the IL-17 antibody-mediated expression levels (Fig. 5B). Subsequently, the results of the TEER assay indicated that treatment with the IL-17 antibody significantly increased the $\mathrm{H} / \mathrm{R}$-mediated reduction in TEER value, and transfection with ov-TAK1 significantly reversed the effects of the IL-17 antibody on the TEER value (Fig. 5C). Furthermore, the expression levels of occludin, claudin-1 and ZO-1 were significantly upregulated in the H/R + IL-17 antibody group compared with the H/R + IgG group, while transfection with ov-TAK1 significantly reduced these expression levels (Fig. 5D).

\section{Discussion}

Intestinal I/R injury is often a synergistic effect of multiple factors (22). Inflammation is a key response to pathogens and, according to a previous report, when intestinal I/R injury occurs, the body stimulates an immune response and simultaneously promotes the upregulation of numerous inflammatory cytokines. Thus, an inflammatory response is aggravated, causing damage to multiple organs (23). Moreover, apoptosis is also considered to be a major mechanism underlying ischemic tissue cell death, which impacts inflammation, calcium overload and oxidation (24). In the process of intestinal I/R, local apoptosis occurs in the intestine, and apoptosis may also be induced in remote organs (25). Notably, the intestinal capillary permeability increases following I/R, which leads to intestinal absorption dysfunction and increased mucosal permeability, allowing macromolecular solutes to pass. Thus, damaged intestines may become the source of numerous harmful biologically active substances (26). Therefore, further investigation into the mechanisms underlying intestinal $\mathrm{I} / \mathrm{R}$ injury is required for the development of novel treatment options.

Results of the present study demonstrated that intestinal I/R causes upregulation of TAK 1 expression, and TAK1 knockdown inhibits the phosphorylation of JNK. Results of a previous study demonstrated that the expression of JNK in the MAPK signaling family is increased in intestinal ischemic tissues (27). Moreover, activation of TAK1-dependent NF- $\kappa \mathrm{B}$ and proto-oncogene c-Jun is associated with the occurrence of intestinal inflammation and, in the case of dextran sodium sulfate-induced enteritis, the expression levels of TAK 1 are significantly increased (28). These findings are consistent with those of the present study. Furthermore, results of the present study also demonstrated that TAK1 was involved in intestinal I/R injury, including cell inflammation, apoptosis and barrier damage. TAK1 knockdown alleviated the inflammation, apoptosis and barrier dysfunction of H/R-induced Caco- 2 cells by inhibiting MAPK signal activation.

Moreover, following intestinal I/R, the release of IL-17 is upregulated. Over the past decade, IL-17 has been revealed to play an important role in autoimmune diseases and tumors (29). At present, an IL-17 monoclonal antibody that has been approved for marketing is used for the treatment of psoriasis (30). However, to the best of our knowledge, there are few studies that describe the role of IL-17 in the intestinal tract. Results of a previous study revealed that IL-17 is upregulated in the gut of mice with intestinal fibrosis (31), and the decrease of IL-17 expression levels may protect intestinal cells from inflammation (32). Results of the present study demonstrated that reducing IL-17 expression alleviated inflammation, apoptosis and barrier dysfunction caused by intestinal I/R injury. Notably, IL-17 exerted the aforementioned effects by reducing the levels of TAK1 expression. Results of a previous study further demonstrated that IL-17 promotes drug efflux in patients with rheumatoid arthritis via regulation of TAK1 (33). Usually, some researchers use recombinant IL-17 to induce inflammation to study the role of IL-17 in cells. In the present article, cells influenced each other through paracrine signaling, therefore, the use of recombinant IL-17 was unnecessary. Therefore, the present study selected to neutralize IL-17 with an antibody to explore its effects.

In conclusion, the present study demonstrated that intestinal I/R induces the release of IL-17 to regulate cell viability, inflammation, apoptosis and barrier damage via 
A

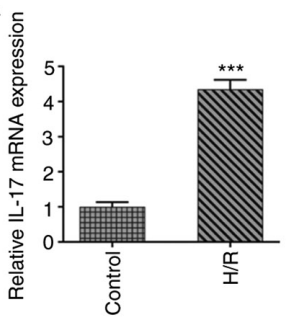

C

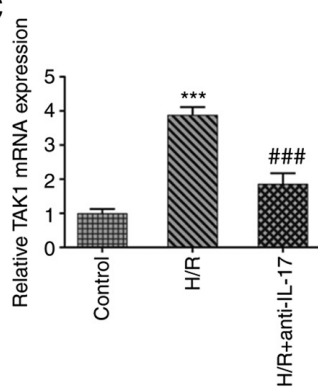

$\mathrm{E}$

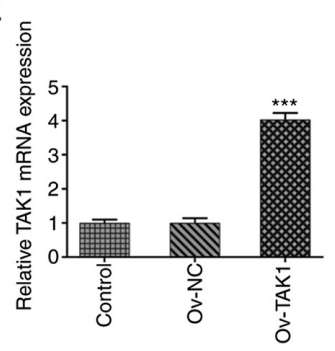

B

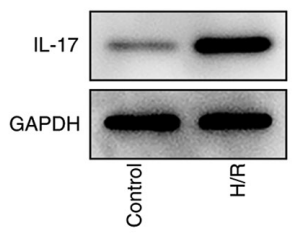

D

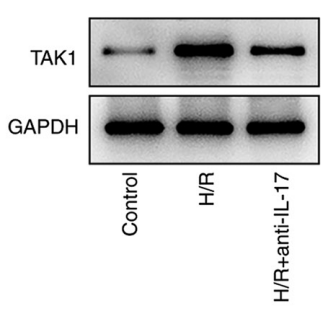

$\mathrm{F}$

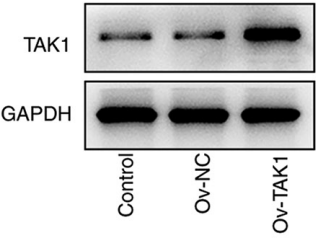

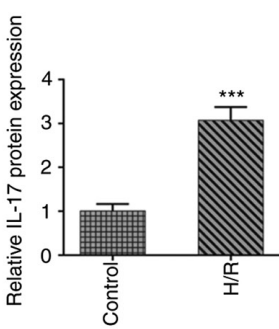
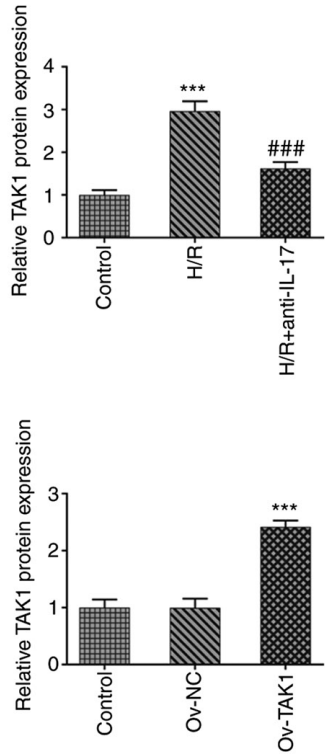

G

$\mathrm{H}$
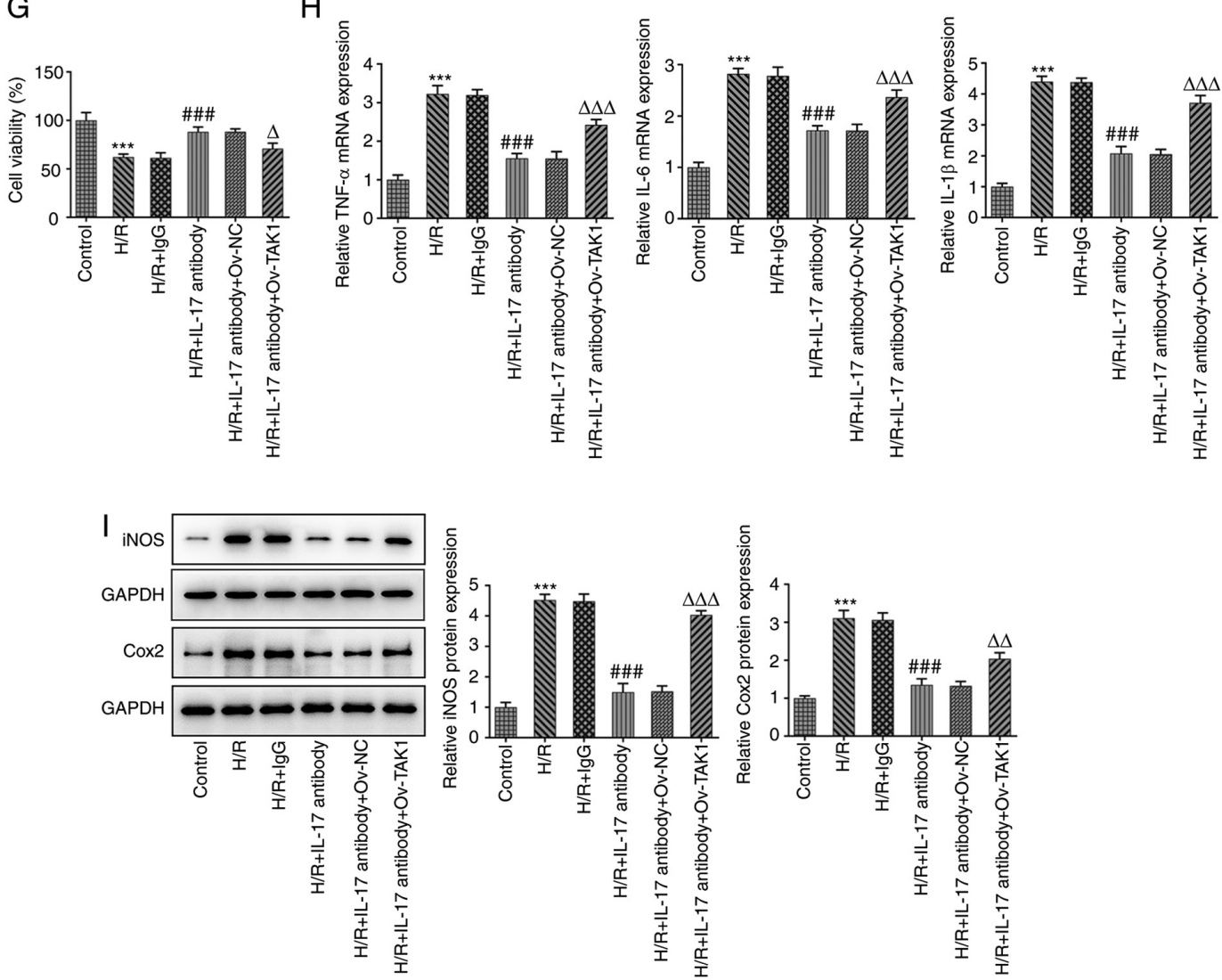

Figure 4. IL-17 antibody improves cell viability and alleviates the inflammation of H/R-induced Caco-2 cells through a reduction of TAK1 expression. Expression levels of IL-17 in the H/R group were determined using (A) RT-qPCR and (B) western blotting. Expression levels of TAK1 were determined using (C) RT-qPCR and (D) western blotting. Expression levels of TAK1 in the transfected cells were determined using (E) RT-qPCR and (F) western blotting. (G) Cell viability in the six groups was measured using a Cell Counting-Kit 8 assay. $(\mathrm{H}) \mathrm{mRNA}$ expression levels of TNF- $\alpha$, IL-6 and IL-1 $\beta$ were determined using RT-qPCR. (I) Protein expression levels of iNOS and Cox 2 were determined using western blot analysis. ${ }^{* * *} \mathrm{P}<0.001 \mathrm{vs}$. control/Ov-NC; ${ }^{\# \# \# ~} \mathrm{P}<0.001 \mathrm{vs}$. $\mathrm{H} / \mathrm{R}+\mathrm{IgG}$; ${ }^{\Delta} \mathrm{P}<0.05$, ${ }^{\Delta \Delta} \mathrm{P}<0.01,{ }^{\Delta \Delta \Delta} \mathrm{P}<0.001$ vs. H/R + IL-17 antibody + Ov-NC. H/R, hypoxia/reoxygenation; RT-qPCR, reverse transcription-quantitative PCR; TAK1, TGF- $\beta$ activated kinase 1; iNOS, inducible nitric oxide synthase; Cox2, cytochrome C oxidase subunit 2; Ov, overexpression; NC, negative control. 
A
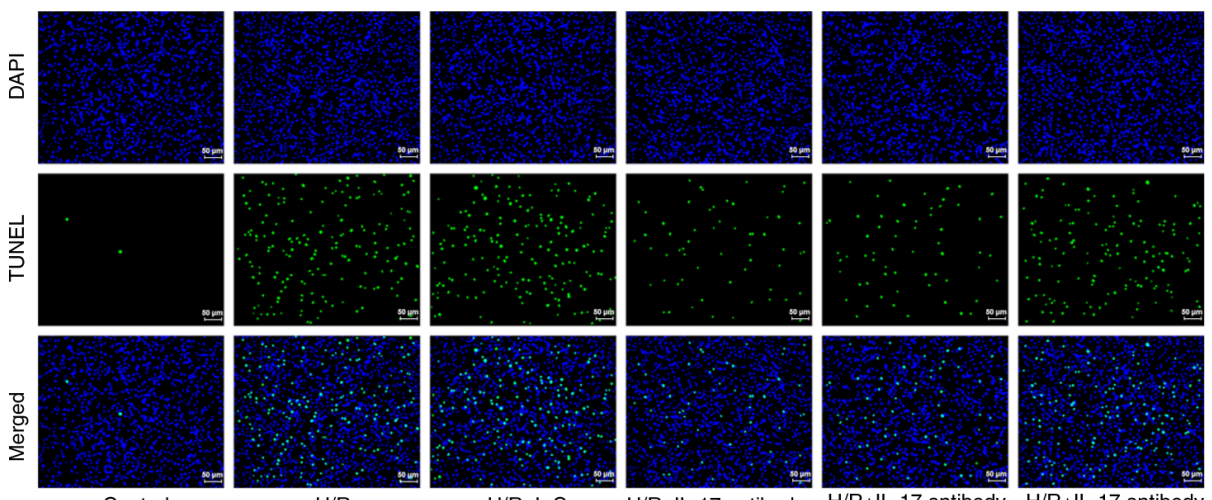

H/R+IL-17 antibody H/R+IL-17 antibody

$+\mathrm{Ov}-\mathrm{NC}$

B

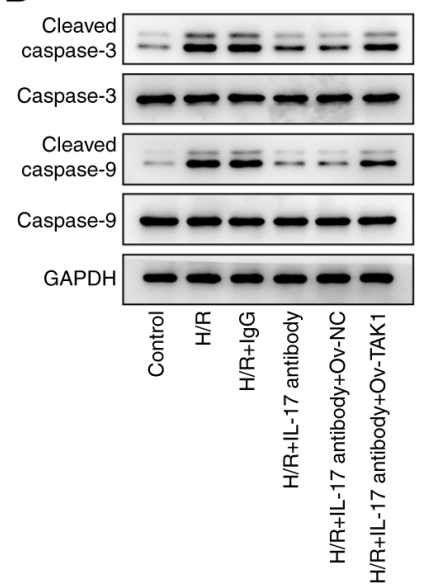

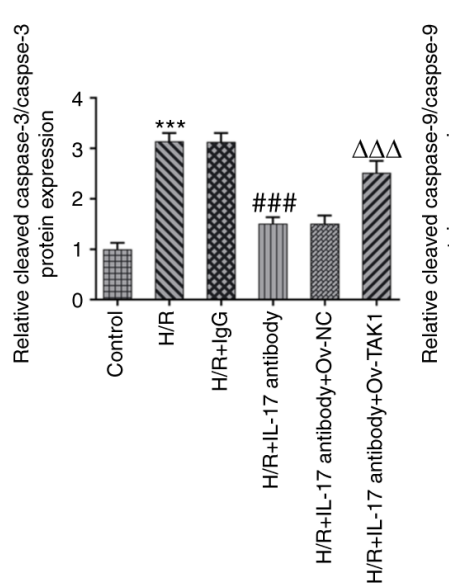

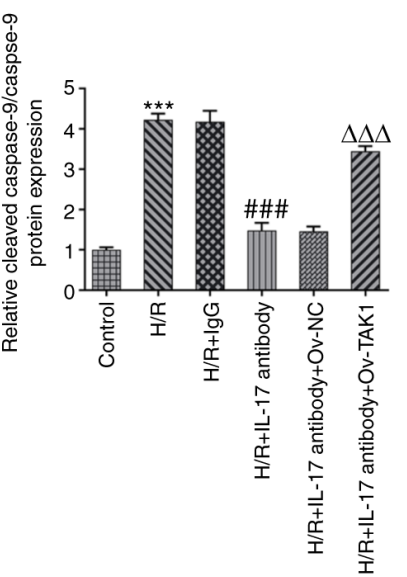

C
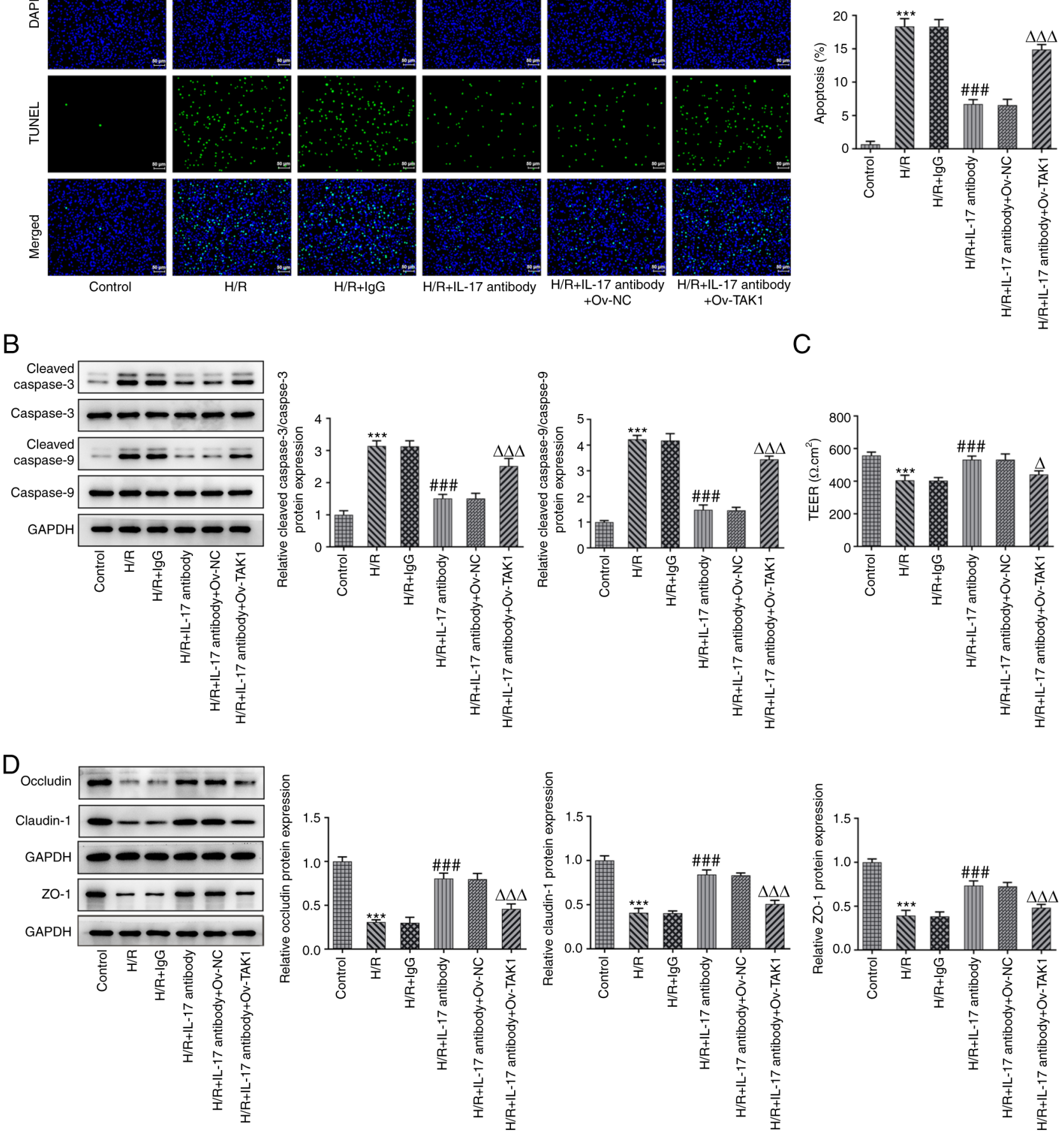

Figure 5. IL-17A antibody alleviates the apoptosis and barrier dysfunction of H/R-induced Caco-2 cells via a reduction in TAK1 expression. (A) Apoptosis was assessed using a TUNEL assay (magnification, x200). (B) Expression levels of cleaved caspase 9 and cleaved caspase 3 were assessed using western blotting. (C) Cell barrier function was assessed using a transepithelial electrical resistance assay. (D) Expression levels of occludin, claudin-1 and ZO-1 were assessed using western blotting. ${ }^{* * *} \mathrm{P}<0.001$ vs. control; ${ }^{\# \# \#} \mathrm{P}<0.001$ vs. $\mathrm{H} / \mathrm{R}+\mathrm{IgG} ;{ }^{\Delta} \mathrm{P}<0.05,{ }^{\Delta \Delta \Delta} \mathrm{P}<0.001$ vs. H/R + IL-17 antibody + Ov-NC. ZO-1, ZO-1 tight junction protein; H/R, hypoxia/reoxygenation; Ov, overexpression; NC, negative control.

TAK1/MAPK signaling. However, the present study is limited to in vitro research, and in vivo intestinal $\mathrm{I} / \mathrm{R}$ models will be established in the future to further explore the roles of IL-17 in I/R injury.

\section{Acknowledgements}

Not applicable.

\section{Funding}

No funding was received.

\section{Availability of data and materials}

The datasets used and/or analyzed during the current study are available from the corresponding author on reasonable request. 


\section{Authors' contributions}

LX and PH conceived the study, and designed and participated in the experiments. LX wrote the manuscript. $\mathrm{PH}$ revised the manuscript. WHZ and YH participated in the experiments and data processing. All the authors have read and approved the final manuscript. LX and $\mathrm{PH}$ confirm the authenticity of the raw data

\section{Ethics approval and consent to participate}

Not applicable.

\section{Patient consent for publication}

Not applicable.

\section{Competing interests}

The authors declare that they have no competing interests.

\section{References}

1. Jozwiak M, Bougouin W, Geri G, Grimaldi D and Cariou A: Post-resuscitation shock: Recent advances in pathophysiology and treatment. Ann Intensive Care 10: 170, 2020.

2. Van Hoof L, Rega F, Devroe S, Degezelle K, Pirenne J and Neyrinck A: Successful resuscitation after hyperkalemic cardiac arrest during liver transplantation by converting Veno-Venous bypass to veno-arterial ECMO. Perfusion 36: 766-768, 2021.

3. Warach SJ, Dula AN and Milling TJ Jr: Tenecteplase thrombolysis for acute ischemic stroke. Stroke 51: 3440-3451, 2020.

4. Fernández AR, Sánchez-Tarjuelo R, Cravedi P, Ochando J and López-Hoyos M: Review: Ischemia reperfusion Injury-A translational perspective in organ transplantation. Int J Mol Sci 21: 8549, 2020.

5. Ali M, Pham A, Wang X, Wolfram J and Pham S: Extracellular vesicles for treatment of solid organ ischemia-reperfusion injury. Am J Transplant 20: 3294-3307, 2020.

6. Zang X, Zhou J, Zhang X, Han Y and Chen X: Ischemia reperfusion injury: Opportunities for nanoparticles. ACS Biomater Sci Eng 6: 6528-6539, 2020.

7. Prieto-Moure B, Cejalvo-Lapeña D, Belda-Antolí M, Padrón-Sanz C, Lloris-Cejalvo JM and Lloris-Carsí JM: Combination therapy of allopurinol and dantrolene and its role in the prevention of experimental ischemia reperfusion injury of the small intestine. J Invest Surg 34: 800-807, 2021.

8. Saleh H and El-Shorbagy HM: Mechanism underlying methyl eugenol attenuation of intestinal ischemia/reperfusion injury. Appl Physiol Nutr Metab 42: 1097-1105, 2017.

9. Tuncer FB, Durmus Kocaaslan FN, Yildirim A, Sacak B, Arabaci Tamer S, Sahin H, Cinel L and Celebiler O: Ischemic preconditioning and Iloprost reduces Ischemia-reperfusion injury in Jejunal flaps: An animal model. Plast Reconstr Surg 144: 124-133, 2019

10. Subramanian S, Geng H and Tan XD: Cell death of intestinal epithelial cells in intestinal diseases. Sheng Li Xue Bao 72 308-324, 2020

11. Zhang X, Wu J, Liu Q, Li X, Li S, Chen J, Hong Z, Wu X, Zhao $\mathrm{Y}$ and Ren J: mtDNA-STING pathway promotes Necroptosis-dependent enterocyte injury in intestinal ischemia reperfusion. Cell Death Dis 11: 1050, 2020.

12. Liu L, Yao J, Li Z,Zu G, Feng D, Li Y, Qasim W, Zhang S, Li T, Zeng H and Tian X: miR-381-3p knockdown improves intestinal epithelial proliferation and barrier function after intestinal ischemia/reperfusion injury by targeting nurrl. Cell Death Dis 9: 411, 2018.

13. Kajino-Sakamoto R, Inagaki M, Lippert E, Akira S, Robine S, Matsumoto K, Jobin C and Ninomiya-Tsuji J: Enterocyte-derived TAK1 signaling prevents epithelium apoptosis and the development of ileitis and colitis. J Immunol 181: 1143-1152, 2008

14. Nighot M, Rawat M, Al-Sadi R, Castillo EF, Nighot P and Ma TY: Lipopolysaccharide-induced increase in intestinal permeability is mediated by TAK-1 activation of IKK and MLCK/MYLK gene. Am J Pathol 189: 797-812, 2019.
15. Lee HT, Kim M, Kim JY, Brown KM, Ham A, D'Agati VD and Mori-Akiyama Y: Critical role of interleukin-17A in murine intestinal ischemia-reperfusion injury. Am J Physiol Gastrointest Liver Physiol 304: G12-G25, 2013.

16. Zepp J, Wu L and Li X: IL-17 receptor signaling and T helper 17-mediated autoimmune demyelinating disease. Trends Immunol 32: 232-239, 2011.

17. Huang F, Kao CY, Wachi S, Thai P, Ryu J and Wu R: Requirement for both JAK-mediated PI3K signaling and ACT1/TRAF6/TAK1-dependent NF-kappaB activation by IL-17A in enhancing cytokine expression in human airway epithelial cells. J Immunol 179: 6504-6513, 2007.

18. Zhou J, An C, Jin X, Hu Z, Safirstein RL and Wang Y: TAK1 deficiency attenuates cisplatin-induced acute kidney injury. Am J Physiol Renal Physiol 318: F209-F215, 2020.

19. Hiebl V, Schachner D, Ladurner A, Heiss EH, Stangl H and Dirsch VM: Caco-2 cells for measuring intestinal cholesterol transport-possibilities and limitations. Biol Proced Online 22: 7 , 2020.

20. Tang LJ, Zhou YJ, Xiong XM, Li NS, Zhang JJ, Luo XJ and Peng J: Ubiquitin-specific protease 7 promotes ferroptosis via activation of the p53/TfR 1 pathway in the rat hearts after ischemia/reperfusion. Free Radic Biol Med 162: 339-352, 2021.

21. Livak KJ and Schmittgen TD: Analysis of relative gene expression data using real-time quantitative PCR and the 2(-Delta Delta C(T)) method. Methods 25: 402-408, 2001.

22. Yang B, Zhang LY, Chen Y, Bai YP, Jia J, Feng JG, Liu KX and Zhou J: Melatonin alleviates intestinal injury, neuroinflammation and cognitive dysfunction caused by intestinal ischemia/reperfusion. Int Immunopharmacol 85: 106596, 2020.

23. Liu DQ, Chen SP, Sun J, Wang XM, Chen N, Zhou YQ, Tian YK and Ye DW: Berberine protects against ischemia-reperfusion injury: A review of evidence from animal models and clinical studies. Pharmacol Res 148: 104385, 2019.

24. Radak D, Katsiki N, Resanovic I, Jovanovic A, Sudar-Milovanovic E, Zafirovic S, Mousad SA and Isenovic ER: Apoptosis and acute brain ischemia in ischemic stroke. Curr Vasc Pharmacol 15: 115-122, 2017.

25. Feng D, Yao J, Wang G, Li Z, Zu G, Li Y, Luo F, Ning S, Qasim W, Chen Z and Tian X: Inhibition of p66Shc-mediated mitochondrial apoptosis via targeting prolyl-isomerase Pin1 attenuates intestinal ischemia/reperfusion injury in rats. Clin Sci (Lond) 131: 759-773, 2017.

26. Li C, Li Q, Liu YY, Wang MX, Pan CS, Yan L, Chen YY, Fan JY and Han JY: Protective effects of Notoginsenoside R1 on intestinal ischemia-reperfusion injury in rats. Am J Physiol Gastrointest Liver Physiol 306: G111-G122, 2014.

27. Ming YC, Chao HC, Chu SM and Luo CC: Heparin-binding epidermal growth factor-like growth factor (HB-EGF) protected intestinal ischemia-reperfusion injury through JNK and p38/MAPK-dependent pathway for anti-apoptosis. Pediatr Neonatol 60: 332-326, 2019.

28. Xu K, Guo Y, Ping L, Qiu Y, Liu Q, Li Z and Wang Z: Protective effects of SIRT6 overexpression against DSS-induced colitis in mice. Cells 9: 1513, 2020 .

29. McGeachy MJ, Cua DJ and Gaffen SL: The IL-17 family of cytokines in health and disease. Immunity 50: 892-906, 2019

30. Ly K, Smith MP, Thibodeaux Q, Reddy V, Liao W and Bhutani T: Anti IL-17 in psoriasis. Expert Rev Clin Immunol 15: 1185-1194, 2019.

31. Li J, Liu L, Zhao Q and Chen M: Role of interleukin-17 in pathogenesis of intestinal fibrosis in mice. Dig Dis Sci 65: 1971-1979, 2020.

32. Prete R, Garcia-Gonzalez N, Di Mattia CD, Corsetti A and Battista N: Food-borne Lactiplantibacillus plantarum protect normal intestinal cells against inflammation by modulating reactive oxygen species and IL-23/IL-17 axis. Sci Rep 10: 16340, 2020.

33. Li Z, Niu H, Yao H, Chen M, Zhao X, Zhao W, Luo J, Gao C, Li X and Wang C: IL-17A upregulates P-glycoprotein expression in peripheral blood lymphocytes of patients with rheumatoid arthritis through TAK1. Clin Exp Rheumatol 38: 299-305, 2020

This work is licensed under a Creative Commons Attribution-NonCommercial-NoDerivatives 4.0 International (CC BY-NC-ND 4.0) License. 